\title{
SHORTFALL RISK MINIMIZATION: THE DUAL APPROACH
}

\author{
Ju Hong Kim
}

\begin{abstract}
We find the solution minimizing the shortfall risk by using the Lagrangemultiplier method. The conventional duality method in the expected utility maximization problem is used and we get the same results as in the paper [21].
\end{abstract}

\section{INTRODUCTION}

We consider an agent or an investor who sell a contingent claim and want to get rid of the associated shortfall risk by means of a dynamic hedging strategy. The shortfall risk is the difference between the payoff of the contingent claim and the value of the agent's or the investor's hedging strategy at maturity.

It is known that there is a dynamic self-financing hedging strategy with arbitragefree hedging price to super-replicate a contingent claim in complete or incomplete markets. The super-hedging price is the minimal initial capital that an agent or an investor has to invest to find a strategy which dominates the claim payoff with certainty [15]. The super-hedging price of a contingent claim is given by the supremum of the expected values over all equivalent martingale measures. If an agent or an investor sells the claim for the super-hedging price, then he/she could eliminate the shortfall risk completely by choosing a suitable hedging strategy. The corresponding value process is a supermartingale under equivalent martingale measures. The super-hedging strategy is determined by the optional decomposition [18]. But the prices derived by super-replication are too high and not acceptable in practice. Then the claim should be sold for a price less than the super-hedging price. With the initial capital less than the super-hedging price, i.e., under the capital constraint

Received by the editors April 5, 2012. Accepted May 15, 2012. 2000 Mathematics Subject Classification. 91B30, 91B24, 91B26.

Key words and phrases. shortfall risk, duality theory, incomplete markets, Fenchel-Legendre transform. 
an agent or an investor is unable to eliminate all exposed risk associated to the contingent claim completely and so wants to find optimal strategies which minimize the shortfall risk.

Föllmer and Leukert [11] constructed a quantile hedging strategy which maximizes the probability of a successful hedge under the objective measure $P$ under the capital constraint. In the quantile hedging approach, the size of the shortfall is not taken into account but only the probability of its occurrence. Föllmer and Leukert [12] also introduced optimal hedging strategies which minimize the shortfall risk under the capital constraint by using the expected loss functions as risk measures. In these papers the Neyman-Pearson lemma approach is used to find the solution to the static problem. In [12], the risk measure $\rho$ is the form of $\rho(X)=E_{P}\left[\ell\left(X^{+}\right)\right]$, where $X$ is a random variable on $(\Omega, \mathcal{F}), P$ is a fixed probability measure on $\Omega$, and $\ell: \mathbb{R} \rightarrow \mathbb{R}$ is a strictly convex function. See the papers $[6,7,23,20]$ for the related works. Nakano [19] uses coherent risk measures [3, 8] as risk measures in the $L^{1}(\Omega, \mathcal{F}, P)$ random variable spaces instead of the loss function. Arai [1] obtained robust representation results of shortfall risk measures on Orlicz hearts under the continuous time setting. The Orlicz hearts setting allows us to treat various loss functions and various claims in a unified framework.

In this paper, we find the solution minimizing the shortfall risk by the dual approach [22]. The conventional duality method used in the expected utility maximization problem is adopted and we get the same results as in [21]. This paper is constructed as follows. The definition of a superhedging price and mathematical settings are given in section 2. The optimal solution of shortfall risk is found in complete market case and in incomplete market case in section 3 and 4, respectively.

\section{Mathematical Settings And Superhedging}

Let $\left(\Omega, \mathcal{F},\left(\mathcal{F}_{t}\right)_{0 \leq t \leq T}, P\right)$ be a complete filtered probability space. Let

$$
S=\left(S_{t}\right)_{0 \leq t \leq T}
$$

be an adapted positive process which is a semimartingale. It is assumed that the riskless interest rate is zero for simplicity.

Definition 2.1. A self-financing strategy with initial capital $x \geq 0$ is defined as a predictable process $\xi_{t}$ such that the value process (value of the current holdings)

$$
X_{t}=x+\int_{0}^{t} \xi_{u} d S_{u}, \quad t \in[0, T]
$$


is $P$-a.s. well-defined.

Definition 2.2. A self-financing strategy $\left(x, \xi_{t}\right)$ is called admissible if there exists some constant $c>0$ such that

$$
\forall t \in[0, T] \quad x+\int_{0}^{t} \xi_{u} d S_{u} \geq-c \quad P-a . s .
$$

Here $c$ is a credit line of an agent or an investor.

Definition 2.3. A contingent claim $H$ is called attainable (or replicable, redundant) if there exists admissible strategy such that

$$
H=x_{0}+\int_{0}^{T} \xi_{u} d S_{u}
$$

Lemma 2.4. Let $H \geq 0$ be a $\mathcal{F}_{T}$-measurable contingent claim. Then there exists admissible strategy $\left(x_{0}, \xi\right)$ such that

$$
H \leq x_{0}+\int_{0}^{T} \xi_{u} d S_{u} \quad P-a . s .
$$

if and only if

$$
H \in\left\{X \geq 0 \mid X \text { is } \mathcal{F}_{T}-\text { measurable, } \quad \sup _{Q \sim P} E_{Q}[X] \leq x_{0}\right\} .
$$

Proof. See the proof in [17].

Lemma (2.4) means that the pricing rule of $H$, i.e., $E_{Q}[H]$ is less than or equal to $x_{0}$ which is the initial capital of the admissible superhedging strategy $\left(x_{0}, \xi\right)$ for $H$.

Definition 2.5. The superhedge price $H_{0}$ for $H$ is defined as

$H_{0}=\inf \left\{x \mid \exists\right.$ admissible strategy $(x, \xi)$ s.t. $\left.H \leq x+\int_{0}^{T} \xi_{u} d S_{u} \quad P-a . s.\right\}$.

By the Lemma (2.4) we can see the superhedge price is $H_{0}=\sup _{Q \sim P} E_{Q}[H]$. That is, $H_{0}$ is the smallest initial capital eliminating all shortfall risk. The seller of $H$ can cover almost any possible obligation from the sale of $H$ and thus eliminate completely the corresponding risk. However, the super-hedging price of the seller is too high and can't be used in practice.

When the seller is unwilling to invest the superhedge price in a hedging strategy, the seller is seeking for the optimal partial hedging strategy minimizing the problem [13] 


$$
\min _{(x, \xi) \in \mathcal{X}(\alpha)}\left[\rho\left(\left(H-x-\int_{0}^{T} \xi_{u} d S_{u}\right)^{+}\right)\right]
$$

with the initial capital constraint

$$
0<\alpha<H_{0}=\sup _{Q \sim P} E_{Q}[H] .
$$

The admissible set $\mathcal{X}(\alpha)$ is defined as

$$
\mathcal{X}(\alpha):=\left\{(x, \xi) \mid x \leq \alpha<H_{0},(x, \xi) \text { is admissible strategy }\right\} .
$$

Hereafter the risk measure $\rho$ is taken as $\rho(X)=E_{P}[\ell(X)]$ as in the traditional literature, where $X$ is a random variable on $(\Omega, \mathcal{F}), P$ is a fixed probability measure on $\Omega$, and $\ell: \mathbb{R} \rightarrow \mathbb{R}$ is a strictly convex function as in [12]. We assume that the function $\ell \in C^{1}(0, \infty)$, the derivative $\ell^{\prime}$ is strictly increasing with $\ell^{\prime}(0+)=0$ and $\ell^{\prime}(+\infty)=+\infty$.

We will often use the short notation $(\xi . S)_{T}$ as the same expression as $\int_{0}^{T} \xi_{u} d S_{u}$. We consider the general set $K(x)$ of the terminal wealths at $T$ with initial wealth $x$, and the set $K(x)$ is defined as

$$
K(x):=\left\{X_{T} \mid X_{T}=x+(\xi . S)_{T} \text { is } \mathcal{F}_{T} \text { - measurable }\right\} .
$$

We can rewrite the minimizing shortfall problem (2.3) as the primal problem

$$
\mathcal{P}(x):=\inf _{X_{T} \in K(x)} E\left[\ell\left(H-X_{T}\right)^{+}\right] .
$$

Define $K_{Q}(x)$ as

$$
K_{Q}(x)=\left\{X \in L^{1}(Q) \mid E_{Q}[X] \leq x\right\},
$$

which contains the set $K(x)$ and the norm-closure of $K(x)-L_{+}^{1}(Q)$ in $L^{1}(Q)$.

The pricing measure $Q$ is unique in complete market but not unique in incomplete market. The set of pricing measures is

$$
\mathcal{M}=\{Q \mid Q \sim P, S \text { is a local martingale under } Q\}
$$

as stated in [9].

Assume that $\mathcal{M} \neq \emptyset$ for the no-arbitrage condition of the markets $[9,10]$.

Note that if $x \geq \sup _{Q \mathcal{M}} E_{Q}[H]:=H_{0}$, then there exist admissible strategies $(x, \xi)$ such that $H \leq H_{0}+(\xi . S)_{T} \leq x+(\xi . S)_{T}$ by the Lemma (2.4) and hence it is hedged completely with the superhedge price.

Assume that $x<\sup _{Q \mathcal{M}} E_{Q}[H]$ throughout this paper. 
2.1. Complete market case. Assume the market is complete and then the pricing measure $Q \in \mathcal{M}$ is unique. We try to solve the primal problem (2.5) under the constraint set $K_{Q}(x)$ which is larger than the set $K(x)$.

Fenchel-Legendre transform or conjugate functional $\ell^{*}$ of the convex function $\ell$ is defined by

$$
\ell^{*}(z):=\sup _{y \in \mathbb{R}}\{y z-\ell(y)\}
$$

Note that $\ell^{*}$ is a proper convex function, i.e. it is convex and takes some finite value. Denote $J:=\left(\ell^{*}\right)_{+}^{\prime}$ its right-continuous derivative. Form (2.6), for all $y, z \in \mathbb{R}$

$$
y z \leq \ell(y)+\ell^{*}(z),
$$

and the equality holds if $y=J(z)$.

We consider the primal problem

$$
\mathcal{P}(x):=\inf _{X_{T} \in K_{Q}(x)} E\left[\ell\left(H-X_{T}\right)^{+}\right] .
$$

When we use the Lagrange-multiplier method [2], we can express the dual problem of the primal one as

$$
\mathcal{D}(x):=\sup _{\lambda>0} \inf _{X_{T} \in L^{1}(Q)}\left\{E\left[\ell\left(H-X_{T}\right)^{+}\right]+\lambda\left(x-E_{Q}\left[X_{T}\right]\right)\right\} .
$$

We will show that there is no duality gap, i.e.

$$
\mathcal{P}(x)=\mathcal{D}(x) .
$$

First we show that for $X_{T}$ satisfying $E_{Q}\left[X_{T}\right] \leq x$

$$
\begin{gathered}
\inf _{X_{T} \in L^{1}(Q)}\left\{E\left[\ell\left(H-X_{T}\right)^{+}\right]+\lambda\left(x-E_{Q}\left[X_{T}\right]\right)\right\} \\
\geq \lambda\left(E_{Q}[H]-x\right)-E\left[\ell^{*}\left(\lambda \frac{d Q}{d P}\right)\right] .
\end{gathered}
$$

If we take $y=\left(H-X_{T}\right)^{+}$and $z=\lambda \frac{d Q}{d P}$ in the equation (2.7), and then take the expectation and add $\lambda\left(x-E_{Q}\left[X_{T}\right]\right)$ to both sides of the inequality, then we have

$$
E\left[\ell\left(H-X_{T}\right)^{+}\right]-\lambda\left(x-E_{Q}\left[X_{T}\right]\right) \geq \lambda\left(E_{Q}[H]-x\right)-E\left[\ell^{*}\left(\lambda \frac{d Q}{d P}\right)\right]
$$

since $E_{Q}[H]>x \geq E_{Q}\left[X_{T}\right]$ and so $E_{Q}\left[\left(H-X_{T}\right)^{+}\right]=\left(E_{Q}[H]-E_{Q}\left[X_{T}\right]\right)^{+}=$ $E_{Q}[H]-E_{Q}\left[X_{T}\right]$. So we have

$$
\mathcal{P}(x):=\inf _{X_{T} \in K_{Q}(x)} E\left[\ell\left(H-X_{T}\right)^{+}\right] \geq \lambda\left(E_{Q}[H]-x\right)-E\left[\ell^{*}\left(\lambda \frac{d Q}{d P}\right)\right] .
$$


The inequality (2.10) holds from (2.11) and the equality in (2.10) holds if for each $\lambda>0$ the relation

$$
\left(H-X_{T}\right)^{+}=\left(\ell^{*}\right)^{\prime}\left(\lambda \frac{d Q}{d P}\right)
$$

satisfies. Hence the dual problem (2.9) becomes

$$
\begin{aligned}
\mathcal{D}(x):= & \sup _{\lambda>0} \inf _{X_{T} \in L^{1}(Q)}\left\{E\left[\ell\left(H-X_{T}\right)^{+}\right]+\lambda\left(x-E_{Q}\left(X_{T}\right)\right)\right\} \\
& \geq \sup _{\lambda>0}\left\{\lambda\left(E_{Q}[H]-x\right)-E\left[\ell^{*}\left(\lambda \frac{d Q}{d P}\right)\right]\right\} .
\end{aligned}
$$

If the equation (2.13) satisfies, then the equality in (2.14) holds. If $E_{Q}\left[X_{T}\right] \leq x$ satisfies in addition to the equation (2.13), then the following relation between the primal and the dual problem holds:

$$
\mathcal{P}(x):=\inf _{X_{T} \in K_{Q}(x)} E\left[\ell\left(H-X_{T}\right)^{+}\right] \geq \mathcal{D}(x) .
$$

For each $\lambda>0$ define

$$
g(\lambda)=\lambda\left(E_{Q}[H]-x\right)-E\left[\ell^{*}\left(\lambda \frac{d Q}{d P}\right)\right] .
$$

Note that for $\lambda \in(0,+\infty)$ the function $g(\lambda)$ is concave function. Under the assumption of $\left(\ell^{*}\right)^{\prime}\left(\lambda \frac{d Q}{d P}\right) \leq h$ for some $h \in L^{1}(P), g$ is differentiable by the Fubini's theorem. Let's find the critical point of $g$.

$$
g^{\prime}(\lambda)=0 \quad \text { if and only if } \quad E_{Q}[H]-x-E_{Q}\left[\left(\ell^{*}\right)^{\prime}\left(\lambda \frac{d Q}{d P}\right)\right]=0 .
$$

It is said that the function $f: \mathbb{R} \rightarrow \mathbb{R}$ has or admits a supporting line at $x \in \mathbb{R}$ if there exists $a \in \mathbb{R}$ such that

$$
f(y) \geq f(x)+a(y-x)
$$

for all $y \in \mathbb{R}$.

Theorem 2.6. If $f$ admits a strict supporting line at $x_{k}$ with slope $k$, then $f^{*}$ admits a tangent supporting line at $k$ with slope $\left(f^{*}\right)^{\prime}(k)=x_{k}$.

Proof. From the Legendre-Fenchel transform

$$
f^{*}(k):=\sup _{x \in \mathbb{R}}\{k x-f(x)\},
$$

we have $f^{*}(k)=k x_{k}-f\left(x_{k}\right)$ and $\left(f^{*}\right)^{\prime}(k)=x_{k}$ where $x_{k}$ is the solution of $f^{\prime}\left(x_{k}\right)=$ $k$. 
Since $\ell: \mathbb{R} \rightarrow \mathbb{R}$ is a strict convex function and the tangent slope of $\ell$ belongs to the range $(0,+\infty)$, the function $\left(\ell^{*}\right)^{\prime}:(0,+\infty) \rightarrow(-\infty,+\infty)$ is bijective by the Theorem (2.6). Hence $\psi(\lambda):=E_{Q}\left[\left(\ell^{*}\right)^{\prime}\left(\lambda \frac{d Q}{d P}\right)\right]$ is a bijective function from $(0,+\infty)$ to $(-\infty,+\infty)$. Thus there exists a unique solution $\lambda^{*}$ of

$$
E_{Q}\left[H-\left(\ell^{*}\right)^{\prime}\left(\lambda \frac{d Q}{d P}\right)\right]=x \text {. }
$$

Therefore, the supremum of the right hand side of (2.14) is taken at $\lambda^{*}$.

Since $x \geq 0, H \geq\left(\ell^{*}\right)^{\prime}\left(\lambda \frac{d Q}{d P}\right) \quad$ Q-a.s.

If we set $X_{T}^{*}=H-\left(\ell^{*}\right)^{\prime}\left(\lambda^{*} \frac{d Q}{d P}\right)$, then $E_{Q}\left[X_{T}^{*}\right]=x$ and so $X_{T}^{*} \in K_{Q}(x)$. By the Lemma (2.4), there exists admissible strategy $(x, \xi)$ satisfying

$$
X_{T}^{*}=x+\int_{0}^{T} \xi_{u} d S_{u} \in K(x) .
$$

Since $H-X_{T}^{*}=\left(\ell^{*}\right)^{\prime}\left(\lambda^{*} \frac{d Q}{d P}\right) \geq 0 \quad Q$-a.s., from Fenchel-Legendre transform we have

$$
\left(\lambda^{*} \frac{d Q}{d P}\right) \cdot\left(H-X_{T}^{*}\right)^{+}=\ell\left(H-X_{T}^{*}\right)^{+}+\ell^{*}\left(\lambda^{*} \frac{d Q}{d P}\right) .
$$

By taking expectation to (2.15) with respect to $P$, we get

$$
E\left[\ell\left(H-X_{T}^{*}\right)^{+}\right]=\lambda^{*}\left(E_{Q}[H]-x\right)-E\left[\ell^{*}\left(\lambda^{*} \frac{d Q}{d P}\right)\right] .
$$

Hence $X_{T}^{*} \in K_{Q}(x)$ is a solution of the primal problem. Thus we are ended up with

$$
\mathcal{P}(x)=\mathcal{D}(x)=\lambda^{*}\left(E_{Q}[H]-x\right)-E\left[\ell^{*}\left(\lambda^{*} \frac{d Q}{d P}\right)\right] .
$$

2.2. Incomplete market case. In this section we adopt notations and the proof methods from $[5,4]$ for the more general approaches. Since equivalent martingale measure is not unique in an incomplete market, the main job is to choose economically suitable one in this subsection, and the rest is the same as in a complete market.

Let $G$ be the convex cone which is a subset of $L^{0}$. Define

$$
\ell_{P, G}(x):=\inf _{X \in G} E_{P}\left[\ell(H-x-X)^{+}\right] .
$$

Define the set $K$ as

$$
K:=\left\{\int_{0}^{T} \xi_{u} \cdot d S_{u} \mid \xi \text { is admissible }\right\},
$$

which is the cone of bounded from below claims that are attainable, at zero initial cost, from trading in the $d$ assets with admissible trading strategies. 
Define $C$ as

$$
C:=\left(K-L_{+}^{0}\right) \cap L^{\infty} .
$$

We know that the norm dual space of $L^{\infty}$ is $b a=b a(\Omega, \mathcal{F}, \mathbb{P})$, the set of bounded additive set functions on $(\Omega, \mathcal{F})$ that are absolutely continuous with respect to $P$.

Let $C^{0}$ be the polar cone of $C$ with respect to the dual system $\left(L^{\infty}, b a\right)$,

$$
C^{0}:=\{\zeta \in b a \mid \zeta(X) \leq 0 \quad \forall X \in C\} .
$$

Definition 2.7. $Q<<P$ is called a separating measure if $K \subseteq L^{1}(Q)$ and if $K \subseteq L^{1}(Q)$ and $E_{Q}[X] \leq 0 \quad \forall X \in K$.

Define $M$ as

$$
M:=C^{0} \cap L^{1}(P)=\left\{z \in L_{+}^{1}(P) \mid E_{P}[z X] \leq 0 \quad \forall X \in C\right\} .
$$

Define the set $M_{1}$ as

$$
M_{1}=\left\{z \in M \mid E_{p}[z]=1\right\} .
$$

A $P$-absolute continuous probability measure $Q$ is identified with its Radon-Nikodym derivative $z=\frac{d Q}{d P}$. So we have

$$
M_{1}=\left\{Q<<P \mid E_{Q}[X] \leq 0 \quad \forall X \in C\right\} .
$$

For all $P \in \mathbb{P}, M \subset L_{+}^{1}(P), M$ is closed in $L^{1}(P)$ and if $M_{1} \neq \emptyset$, the convex cone $M$ is generated by the convex set $M_{1}$.

Lemma 2.8. $Q$ is a separating measure if and only if $Q \in M_{1}$. If $S$ is bounded, then $M=\{Q<<P \mid X$ is a $Q$ - martingale $\}$. If $S$ is locally bounded, then $M=$ $\{Q<<P \mid S$ is a $Q-$ local martingale $\}$.

Proof. It is clear that $M \subset M_{1}$. Conversely, suppose that $Q \in M_{1}$. Let $X \in K$ and set $X_{n}=\min \{X, n\}$. Then $X_{n}=X-\left(X-X_{n}\right) \in C$, and $X_{n} \uparrow X P$-a.s., and hence $Q$-a.s.. By the Lebesgue Dominated Convergence Theorem, $E_{Q}[X]=$ $\lim _{n \rightarrow \infty} E_{Q}\left[X_{n}\right] \leq 0$. Hence $Q$ is a separating measure. If $S$ is bounded and $S=$ $1_{A}\left(S_{t}-S_{s}\right), A \in \mathcal{F}_{s}, 0 \leq s<t \leq T$, then $S \in K$ and $-S \in K . Q \in M_{1}$ implies $E_{Q}\left[1_{A}\left(S_{t}-S_{s}\right)\right]=0$ and $Q$ is a martingale measure.

Define $\ell_{P, K}(x)$ as

$$
\begin{aligned}
\ell_{P, K}(x) & :=\inf _{X \in K} E_{P}\left[\ell(H-x-X)^{+}\right] \\
& =\inf _{\xi \in \mathcal{X}(x)} E_{P}\left[\ell\left(H-x-(\xi . S)_{T}\right)^{+}\right] \quad \text { with } E_{P}[\ell(H+c)]<+\infty,
\end{aligned}
$$

where $-c$ is the credit line of the investors. 
Note that $0 \leq \ell_{P, K}(x)<+\infty$.

Delbaen and Schchermayer [10] showed that NFLVR (no free lunch with vanishing risk) : $\bar{C} \cap L_{+}^{\infty}=\{0\}$, which is the weak no-arbitrage condition of the market, is equivalent to $M_{1} \cap \mathbb{P} \neq \emptyset$. Here $\bar{C}$ is the $L^{\infty}$-norm closure of $C$.

Assume that $M_{1} \cap \mathbb{P} \neq \emptyset$ hereafter.

Note that if $x \geq \sup _{Q \in M_{1}} E_{Q}[H]:=H_{0}$, then there exists an admissible strategy $\xi$ satisfying $H \leq H_{0}+(\xi . S)_{T} \leq x+(\xi . S)_{T}$ by the Lemma (2.4) and hence $\ell_{P, K}(x)=0$. For each $\zeta \in b a$ define $\zeta(X)=E_{P}[\zeta X]$ for all $X \in C$.

Lemma 2.9. If $M_{1} \cap \mathbb{P} \neq \emptyset$, then we have

$$
C=M^{0} \text {. }
$$

Proof. From the definition of polar cone of $C$, we have

$$
\begin{aligned}
C^{0} & =\{\zeta \in b a \mid \zeta(X) \leq 0 \quad \forall X \in C\} \\
& =\left\{\zeta \in b a \mid E^{P}[\zeta X] \leq 0 \quad \forall X \in C\right\}=M .
\end{aligned}
$$

Since $N F L V R$ implies that $C$ is weak*-closed, by the bipolar theorem we have

$$
M^{0}=C^{00}=C \text {. }
$$

The above lemma is adopted from the paper [4].

Lemma 2.10. The following equality holds.

$$
\ell_{P, K}(x)=\ell_{P, C}(x)=\ell_{P, M^{0}}(x) .
$$

Proof. By (2.9), the second equality hold. For the proof of the first equality, let $X \in K$ and $X_{n} \in \min \{X, n\}$. Then $X_{n} \uparrow X$ and $X_{n} \in L^{\infty}$. Since $X_{n}=X-$ $\left(X-X_{n}\right) \in K-L_{+}^{0}, X_{n} \in C$. Moreover, since $H-x-X_{n} \downarrow H-x-X$ and $\ell\left(H-x-X_{n}\right) \downarrow \ell(H-x-X)$, by Lebesgue Dominated Convergence Theorem, $E_{P}\left[\ell\left(H-x-X_{n}\right)\right] \downarrow E_{P}[\ell(H-x-X)]$. Therefore, we have

$$
\ell_{P, C}(x)=\inf _{X \in C} E_{P}\left[\ell(H-x-X)^{+}\right] \leq \inf _{X \in K} E_{P}\left[\ell(H-x-X)^{+}\right]=\ell_{P, K}(x) .
$$

On the other hand, we have

$$
\begin{aligned}
\inf _{X \in C} E_{P}\left[\ell(H-x-X)^{+}\right] & =\inf _{X \in K-L_{+}^{0}} E_{P}\left[\ell(H-x-X)^{+}\right] \\
& \geq \inf _{X \in K} E_{P}\left[\ell(H-x-X)^{+}\right] .
\end{aligned}
$$

Thus the proof is done. 
For $Q<<P$, define

$$
\begin{aligned}
\ell(x ; Q, P) & :=\inf \left\{E_{P}\left[\ell(H-x-X)^{+}\right] \mid X \in L^{\infty}, E_{Q}[X] \leq 0\right\} \\
& =\inf \left\{E_{P}\left[\ell(H-x)^{+}\right] \mid X \in L^{\infty}, E_{Q}[X] \leq x\right\} .
\end{aligned}
$$

Since $M_{1} \neq \emptyset$ by assumption and $M$ is generated by the convex set $M_{1}$, the polar cone $M^{0}$ can be expressed as

$$
M^{0}=\left\{X \in L^{\infty} \mid E_{Q}[X] \leq 0 \forall Q \in M_{1}\right\} .
$$

Note that

$$
\ell_{P, M^{0}}(x) \leq \ell(x ; Q, P),
$$

since $\ell_{P, M^{0}}(x)=\inf \left\{E_{P}\left[\ell(H-X)^{+}\right] \mid X \in L^{\infty}, E_{Q}[X] \leq x \forall Q \in M_{1}\right\}$.

Definition 2.11. $\hat{Q}_{x}$ is called a minimax measure if

$$
\ell_{P, M^{0}}(x)=\min _{Q \in M_{1}} \ell(x ; Q, P)=\ell\left(x ; \hat{Q}_{x}, P\right) .
$$

If there exists $Q^{*} \in M_{1}$ such that $\ell_{P, M^{0}}(x) \neq \ell\left(x ; Q^{*}, P\right)$, then

$$
\ell_{P, K}(x)=\ell_{P, M^{0}}(x)<\ell\left(x ; Q^{*}, P\right) \leq \inf \left\{E_{P}\left[\ell(H-x)^{+}\right] \mid X \in L^{\infty}, E_{Q}[X] \leq x\right\} .
$$

That is,

$$
\inf _{\xi \in \mathcal{X}(x)} E_{P}\left[\ell\left(H-x-(\xi . S)_{T}\right)^{+}\right]<\inf \left\{E_{P}\left[\ell(H-X)^{+}\right] \mid X \in L^{\infty}, E_{Q^{*}}[X] \leq x\right\},
$$

which is economically unreasonable.

Define the indicator functional of a convex set $F \subset L^{\infty}$ with

$$
\delta(w \mid F):=\left\{\begin{array}{cc}
0 & w \in F, \\
+\infty & w \notin F .
\end{array}\right.
$$

The convex conjugate $\delta_{F}^{*}: b a \rightarrow \mathbb{R}$ is denoted by

$$
\delta_{F}^{*}(\zeta):=\sup _{w \in F}\{\zeta(w)-\delta(w)\}=\sup _{w \in F} \zeta(w) .
$$

Let $g: L^{\infty} \rightarrow \mathbb{R}$ be

$$
g(X)=\delta(X \mid G+H-x) .
$$

Define the convex integral functional $I_{u}: L^{\infty} \rightarrow \mathbb{R}$ as

$$
I_{\ell}(X):=E_{P}\left[\ell(X)^{+}\right] .
$$

Lemma 2.12. Suppose that $\ell: \mathbb{R} \rightarrow \mathbb{R}$ is a convex function and that $G$ is a convex cone. Then

$$
\left.\ell_{P, G}(x):=\inf _{X \in x+G} E_{P}\left[\ell(H-X)^{+}\right]\right\}=\min _{\zeta \in G^{0}}\left\{g^{*}(\zeta)-I_{\ell}^{*}(\zeta)\right\} .
$$


Proof. Let's consider the dual expression $g^{*}$ of $g$.

$$
\begin{aligned}
& g^{*}(\zeta)=\sup _{X \in G+H-x} \zeta(X)=\zeta(H-x)+\sup _{X \in G} \zeta(X)=\left\{\begin{array}{cc}
\zeta(H-x) & X \in G^{0} \subset b a, \\
+\infty & \text { otherwise }
\end{array} .\right. \\
& \ell_{P, G}(x)=\inf _{X \in G} E_{P}\left[\ell(H-x-X)^{+}\right]=\inf _{X \in G+H-x} E_{P}\left[\ell(X)^{+}\right] \\
&=\inf _{X \in L^{\infty}}\left\{E_{P}\left[\ell(X)^{+}\right]-\delta(X \mid G+H-x)\right\} \\
&=\max _{\zeta \in G^{0}}\left\{g^{*}(\zeta)-I_{\ell}^{*}(\zeta)\right\},
\end{aligned}
$$

by the Fenchel duality theorem.

Proposition 2.13. Suppose that $\ell: \mathbb{R} \rightarrow \mathbb{R}$ is a convex function and that $G$ is a convex cone with $L_{-}^{\infty} \subset G \subset L^{\infty}$, and $N \subset L_{+}^{1}(P)$ is not an empty convex cone $\sigma\left(b a, L^{\infty}\right)$-closed, $G$ is defined as

$$
G:=N^{0}=\left\{w \in L^{\infty} \mid E^{P}[z w] \leq 0 \quad \forall z \in N\right\} .
$$

Then $G^{0}=N$ and

$$
\ell_{P, G}(x)=\max _{z \in N} E_{P}\left[z(H-x)-\ell^{*}(z)\right] .
$$

Proof. Since $N \subset L_{+}^{1}(P)$ is $\sigma\left(b a, L^{\infty}\right)$-closed, by the bipolar theorem $G^{0}=N^{00}=$ $N$.

If $z \in G^{0} \subset L_{+}^{1}(P)$, then $g^{*}(z)=z(H-x)=E_{P}[z(H-x)]$. Hence we have

$$
\begin{aligned}
\ell_{P, G}(x) & =\max _{\zeta \in G^{0}}\left\{g^{*}(\zeta)-I_{\ell}^{*}(\zeta)\right\} \\
& =\max _{z \in N} E_{P}\left[z(H-x)-\ell^{*}(z)\right] .
\end{aligned}
$$

Corollary 2.14. Suppose that $\ell: \mathbb{R} \rightarrow \mathbb{R}$ is a convex function, $Q<<P$, and that $\ell(x ; Q, P)>\inf _{y \in \mathbb{R}} \ell(y)$. Then

$$
\begin{aligned}
\ell(x ; Q, P) & :=\inf \left\{E_{P}\left[\ell(H-x-X)^{+}\right] \mid X \in L^{\infty}, E_{Q}[X] \leq 0\right\} \\
& =\inf \left\{E_{P}\left[\ell(H-X)^{+}\right] \mid X \in L^{\infty}, E_{Q}[X] \leq x\right\} \\
& =\max _{\lambda \in(0,+\infty)}\left\{\lambda\left(E_{Q}[H]-x\right)-E_{P}\left[\ell^{*}\left(\lambda \frac{d Q}{d P}\right)\right]\right\} .
\end{aligned}
$$

Proof. Let $Q$ be given. Set

$$
N=\left\{z \in L_{+}^{1}(P) \mid z=\lambda \frac{d Q}{d P}, \lambda \geq 0\right\} .
$$


Then

$$
G:=N^{0}=\left\{X \in L^{\infty} \mid E[z X] \leq 0 \forall z \in N\right\}=\left\{X \in L^{\infty} \mid E_{Q}[X] \leq 0\right\},
$$

and so by definition

$$
\ell(x ; Q, P)=\ell_{P, G}(x) .
$$

Hence we have

$$
\begin{aligned}
\ell(x ; Q, P) & =\ell_{P, G}(x)=\max _{z \in N} E_{P}\left[z(H-x)-\ell^{*}(z)\right] \\
& =\max _{\lambda \in[0,+\infty)}\left\{\lambda\left(x-E_{Q}[H]\right)-E_{P}\left[\ell^{*}\left(\lambda \frac{d Q}{d P}\right)\right]\right\} .
\end{aligned}
$$

If $\lambda=0$, then

$$
\ell(x ; Q, P)=E_{P}\left[-\ell^{*}(0)\right]=-\ell^{*}(0)=-\sup _{y \in \mathbb{R}}\{y \cdot 0-\ell(y)\}=\inf _{y \in \mathbb{R}} \ell(y) .
$$

So $\lambda=0$ is excluded.

\section{Lemma 2.15 .}

$$
\begin{gathered}
M=\left\{Q<<P \mid E_{Q}[X] \leq 0 \forall X \in C\right\} . \\
\inf \left\{E_{P}\left[\ell(H-x-X)^{+}\right] \mid X \in K\right\}=\inf \left\{E\left[\ell(H-x-X)^{+}\right] \mid X \in C\right\} .
\end{gathered}
$$

Proof. Let $X \in K$ and

$$
X_{n}:=\min \{X, n\} \in X-\left(X-X_{n}\right) \in\left(K-L_{+}^{0}\right) \cap L^{\infty}(P):=C .
$$

Then $X_{n} \uparrow X \quad P$-a.s. and $0 \geq \lim E_{Q}\left[X_{n}\right]=E_{Q}[X]$ by Monotone Convergence Theorem. The right hand set includes $M$. The other inclusion is obvious by the definition of $C$.

Theorem 2.16. $M \neq \emptyset$ and there exists $Q_{x} \in M$ that satisfies

$$
\inf \left\{E_{P}\left[\ell(H-x-X)^{+}\right] \mid X \in C\right\}=\ell_{P, G}(x)=\max _{Q \in M} \ell(x ; Q, P)=\ell\left(x ; Q_{x}, P\right) .
$$

Proof. If $X \in C$, then $X \leq 0$ and so $E_{P}[X] \leq 0$. Therefore $P \in M \neq \emptyset$.

$$
\begin{aligned}
\ell_{P, G}(x) & =\max _{z \in N, z \neq 0} E_{P}\left[z(H-x)-\ell^{*}(z)\right] \\
& =\max _{Q \in M_{1}}\left\{\max _{\lambda \in(0,+\infty)}\left\{\lambda\left(x-E_{Q}[H]\right)-E_{P}\left[\ell^{*}\left(\lambda \frac{d Q}{d P}\right)\right]\right\}\right\} \\
& =\max _{Q \in M_{1}} \ell(x ; Q, P) .
\end{aligned}
$$




\section{REFERENCES}

1. T. Arai: Good deal bounds induced by shortfall risk. preprint (2009).

2. M. Avriel: Nonlinear Programming: Analysis and Methods. Dover Publications, Inc., New York, 2003.

3. P. Artzner, F. Delbaen, J.-M, Eber \& D. Heath: Coherent measures of risk. Mathematical Finance 9(1999), 203-223.

4. F. Bellini \& M. Fritelli: Utility maximization in incomplete markets for unbounded processes. Mathematical Finance 21 (2002), 1-21.

5. __ : On the existence of minimax martingale measures. Finance Stoch. 9 (2005), 493-517.

6. J. Cvitanic: Minimizing expected loss of hedging in incomplete and constrained markets. SIAM J. Control Optim. 38 (2000), 1050-1066.

7. J. Cvitanic \& I. Karatzas: On dynamic measures of risk. Finance Stoch. 3 (1999), 451-482.

8. F. Delbaen: Coherent risk measures on general probability spaces. Advances in finance and stochastics: Essays in honor of Dieter Sondermann (2002), Springer, 1-37.

9. F. Delbaen \& W. Schachermayer: A General version of the fundamental theorem of asset pricing. Mathematische Annalen 300 (1994), 463-520.

10. _ : The fundamental theorem of asset pricing for unbounded stochastic processes. Mathematische Annalen 312 (1994), 463-520.

11. H. Föllmer \& P. Leukert: Quantile hedging. Finance and Stochastics 10 (1999), 215250 .

12. __ Efficient hedging: Cost versus shortfall risk. Finance and Stochastics 4 (2000), $117-146$.

13. H. Föllmer \& A. Schied: Stochastic Finance: An Introduction in Discrete Time. Springer-Verlag, New York, 2002.

14. I. Karatzas \& S.E. Shreve: Brownian Motion and Stochastic Calculus. Springer-Verlag, New York, 1991.

15. N. El Karoui \& M.C. Quenez: Dynamic programming and pricing of contingent claims in an incomplete market. SIAM J. Control and Optimization 33 (1995), 29-66.

16. I. Karatzas, S.E. Shreve, J. Lehoczky \& G. Xu: Martingale and duality methods for utility maximization in an incomplete market. SIAM J. Control and Optimization 29 (1991), 702-730.

17. Ju Hong Kim: Optimal partial hedging coherent measure of risk. J. Appl. Math. \& Informatics 29 (2011), 987-1000.

18. D.O. Kramkov: Optional decomposition of supermartingales and hedging contingent claims in an incomplete security market. Probability Theory and Related Fields 105 (1996), 459-479. 
19. Y. Nakano: Efficient hedging with coherent risk measures. J. Math. Anal. Appl. 293 (2004), 345-354.

20. H. Pham: Dynamic $L^{P}$-hedging in descrete-time under cone constraints. SIAM J. Control and Optimization 38 (2000), 665-682.

21. __ Minimizing shortfall risk and applications to finance and insurance problems. Annals of Applied Probability 12 (2002), 143-172.

22. R.T. Rockfellar: Conjugate Duality and Optimization. Conference Board of Math. Sciences Series, SIAM Publications, No. 16, 1974.

23. G. Spivak \& J. Cvitanic: Maximizing the probability of a perfect hedge. Annals of Applied Probability 9 (2002), 1303-1328.

Department of Mathematics, Sungshin Women's University, Seoul 136-742, Korea

Email address: jhkkim@sungshin.ac.kr 